\title{
Inhibiting tumor growth of colorectal cancer by blocking the expression of vascular endothelial growth factor receptor 3 using interference vector-based RNA interference
}

\author{
ZHICHENG LUI ${ }^{1,2}$, QIANG MA ${ }^{3}$, XIANGHUI WANG $^{2}$ and YOUCHENG ZHANG ${ }^{1}$ \\ ${ }^{1}$ Department of General Surgery, Lanzhou University Second Hospital, Lanzhou 730030; \\ ${ }^{2}$ Department of Oncology; ${ }^{3}$ Department of Gastro-intestine, Lanzhou Regime General Hospital of PLA, \\ Lan Zhou 730000, P.R. China
}

Received July 29, 2009; Accepted August 31, 2009

DOI: 10.3892/ijmm_00000313

\begin{abstract}
Many reports show that vascular endothelial growth factor receptor 3 (VEGFR3) plays an essential role in tumor metastasis and is a promising target for cancer therapy. The present study was designed to determine the role of VEGFR3 in tumor growth using RNA interference (RNAi) technology. Three small interfering RNA (siRNA) sequences for the VEGFR3 gene were cloned into expression plasmids (pSUPER) and transfected into human colorectal carcinoma (CRC) LoVo cells. Stable transfection of these plasmids decreased VEGFR3 protein expression, leading to the potent suppression of tumor cell proliferation and lymphangiogenesis in vitro. Furthermore, we selected the most effective silenced expressor vector and injected it and pSUPER vector into a tumor xenograft model in nude mice. The tumor growth of LoVo cells expressing VEGFR3 siRNA were significantly inhibited compared with cells transfected with control vector alone. Immunohistochemical analyses of tumor sections revealed a decreased vessel density and decreased VEGFR3 expression in animals when siRNA against VEGFR3 was expressed. These results showed that RNAi of VEGFR3 is an effective tool to reduce lymphangiogenesis in CRC.
\end{abstract}

\section{Introduction}

Colorectal carcinoma (CRC) is the third leading cause of cancer in both men and women and accounts for $\sim 10 \%$ of all new cancer cases and cancer deaths (1). Whereas the overall 5 -year survival rate for patients with CRC is 64\%, the rate drops to $\leq 10 \%$ in patients with a metastatic disease (1). By the time

Correspondence to: Professor You Cheng Zhang, Department of General Surgery, Lanzhou University Second Hospital, Lanzhou 730030, P.R. China

E-mail: youchengzhang@sohu.com

Key words: colorectal cancer, proliferation, RNA interference, vascular endothelial growth factor receptor 3 of diagnosis, $19 \%$ of CRC cases are metastatic. In fact, when cancer is diagnosed, not only colorectal cancer, but for most malignant cancers, metastatic foci appear, being frequently the first symptom. Lymph node metastasis, a frequent occurrence in the early stages of many types of cancer metastases, is considered as a useful prognostic indicator. For most types of cancer, the first site of metastasis is the lymph nodes, and the extent of lymph node involvement is a major criterion for evaluating patient prognosis and the choice of therapy $(2,3)$. Many experimental studies have indicated that lymphangiogenic factor receptor (VEGFR3) and its ligands (VEGF-C and -D) stimulate lymphangiogenesis in tumors and induce proliferation and growth of new lymphatic capillaries, and then enhance the incidence of lymph node metastasis (4-7). Interfering with the VEGF-C/ VEGFR3 signaling pathway was suggested to be a useful clinical strategy in the treatment of lymphatic metastasis $(4,6,8-10)$. All studies show that VEGFR3 is an important trigger of metastasis of tumors. Our aim was to determine whether down-regulated expression of VEGFR3 reduces lymphangiogenesis and inhibit primary tumor growth.

RNA interference (RNAi) is the sequence-specific, posttranscriptional gene-silencing method initiated by doublestranded RNA that are homologous to the gene being suppressed. The high efficiency and specificity of RNAmediated interference has made it a powerful and widely used tool for the analysis of gene function. Considering that VEGFR3 expression is up-regulated in the tumor (11-13), we used a vector-based VEGFR3 siRNA expression system to suppress the expression of VEGFR3 in LoVo human CRC cells and to evaluate its therapeutic efficacy of inhibiting primary tumor growth and lymph node metastasis.

\section{Materials and methods}

Cell culture. The human colon carcinoma cell line, LoVo cells, a human colorectal adenocarcinoma cell line, was obtained from the Shanghai Institutes for Biological Sciences of the Chinese Academy of Sciences. The cells were grown in Dulbecco modified Eagle's medium (Invitrogen, USA), supplemented with $10 \%$ fetal bovine serum (FBS, Invitrogen), $50 \mathrm{U} / \mathrm{ml}$ penicillin and $50 \mu \mathrm{g} / \mathrm{ml}$ streptomycin. 
LoVo cells were maintained in a humidified $37^{\circ} \mathrm{C}$ incubator with $5 \% \mathrm{CO}_{2}$, fed every 3 days with complete medium containing $10 \% \mathrm{FBS}$, and sub-cultured when confluence was reached.

Generation of VEGFR3 siRNA expression plasmids and identification. We selected siRNA sequences as reported by Elbashir et al (14). We used the following procedure to design the VEGFR3 siRNA: i) searched for sequences 5'-AA(N19) or 5 '-NA(N19), where $\mathrm{N}$ is any nucleotide in the intended mRNA sequence, and used only those sequences that occur within an open reading frame, preferably 50-100 nt downstream of the start codon, and show 47 or $52 \% \mathrm{G} / \mathrm{C}$ content; ii) performed a BLAST search with the selected siRNA sequences against expressed sequence tag libraries to ensure that only a single gene is targeted; and iii) searched for any predicted secondary structure of the target mRNA that might inhibit siRNA binding. Three siRNA targeting human VEGFR3 with the following sense and antisense sequences were used: VEGFR3 siRNA1, 5'-CATCACGGAGGAGTCACAC-3' (sense) and 5'-GTGTGACTCCTCCGTGATG-3' (antisense); VEGFR3 siRNA2, 5'-GTACATCAAGGCACGCATC-3' (sense) and 5'-GATGCGTGCCTTGATGTAC-3' (antisense); and VEGFR3 siRNA3, 5'-GGGCAGAATCATCACGAAG-3' (sense) and 5'-CTTCGTGATGATTCTGCCC-3' (antisense). All siRNA were synthesized by Shanghai Biotechnology Co., Ltd., Shanghai, China. Sense and antisense primers, which contain the sense siRNA, 9 bp loop, antisense siRNA, and RNA polymerase III terminator sequences, were created with Bgl and Hind III restriction sites on the $3^{\prime}$ and $5^{\prime}$ ends, respectively. These primers were annealed and inserted into pSUPER vectors, which were kindly provided by Yvonne D. Krom (Department of Human Genetics, Leiden University Medical Center, The Netherlands), following the manufacturer's instructions. The resultant plasmids containing siRNA sequences 1, 2, and 3 were named pSUPER-siRNA1/VEGFR3, pSUPER-siRNA2/VEGFR3, and pSUPER-siRNA3/VEGFR3, respectively. These recombinant vectors were identified by digesting with EcoRI and Hind III.

Transfection of colon tumor cells. LoVo cells $(50,000)$ were seeded into each well in six-well issue culture plates and grown overnight. The medium was replaced with complete medium without fetal bovine serum (FBS). The recombinant pSUPERsiRNA/VEGFR3 and the empty pSUPER were transfected into LoVo cells using Lipofectamine 2000 (Invitrogen) according to the manufacturer's instructions. The medium was replaced with a fresh medium of calf serum $(150 \mathrm{ml} / \mathrm{l})$ after $5 \mathrm{~h}$ transfection. Transfected cells were selected with $400 \mu \mathrm{g} / \mathrm{ml}$ of G418-sulfate. Stably transfected LoVo-siRNA1/VEGFR3, LoVo-siRNA2/VEGFR3, LoVo-siRNA3/VEGFR3 and LoVo-pSUPER control cells were obtained. Cells were harvested at different times points. Western blotting, qRT-PCR analysis and other experiments were performed.

MTT assay. Cell proliferation was measured by the MTT assay. LoVo-siRNA1/VEGFR3, LoVo-siRNA2/VEGFR3, LoVosiRNA3/VEGFR3 and LoVo-pSUPER cells control were seeded into $38-\mathrm{mm}^{2}$ wells of 96-well flat-bottom plates in triplicate and allowed to adhere overnight. MTT (200 $\mu 1$ of
Table I. Sequence of primers and amplified length of genes.

\begin{tabular}{llc}
\hline Gene & \multicolumn{1}{c}{ Sequence } & $\begin{array}{c}\text { Amplified } \\
\text { length (bp) }\end{array}$ \\
\hline VEGFR3 & 5'-CGGGGAAGGG GAGGGAGGAG-3' & $224 \mathrm{bp}$ \\
& 5'-GAAAGGCGGCGGGTGTCAGG-3 & \\
GAPDH & 5'-TAAGTATGACTCCACCCACG-3' & $387 \mathrm{bp}$ \\
& 5'-CTAGCACCTTCCCAACTA-3' & \\
\hline
\end{tabular}

$5 \mathrm{mg} / \mu 1$ solution) (Sigma, Guangzhou, China) in PBS was added to each well. The plates were then incubated for $4 \mathrm{~h}$ at $37^{\circ} \mathrm{C}$. Following 24,48 , and $72 \mathrm{~h}$ of culture, proliferative activity was determined by the 3-(4,5-dimethylthiazol-2-yl)-2,5 bromide assay using a microplate reader at a wavelength of $492 \mathrm{~nm}$. All experiments were done in quadruplicate.

Flow cytometry. Cells were collected by trypsinization and prepared as a single cell suspension by mechanical blowing with PBS, washed with cold PBS twice, and fixed with $70 \%$ alcohol at $4^{\circ} \mathrm{C}$ for $24 \mathrm{~h}$. Fixed cells were washed with PBS and treated with RNase A (Sigma), stained with propidium iodide (Sigma) for $30 \mathrm{~min}$ at room temperature in the dark. DNA content in propidium iodide-stained cells was detected by FCM.

Real-time quantitative RT-PCR analysis. Total cellular RNA was isolated from LoVo-pSUPER control and LoVo-siRNA1/ VEGFR3, LoVo-siRNA2/VEGFR3, LoVo-siRNA3/VEGFR3 cells using Trizol reagent (Invitrogen), quantified, and integrity was tested by gel electrophoresis. As shown in Table I, specific primers for VEGFR3 and GAPDH genes were designed. Primers were purchased from Shanghai Biological Engineering. PCR reactions were performed in LightCycler apparatus (Roche Inc.). The reactions were set up in microcapillary tubes using an LC Fast Start DNA SYBR Green I Kit (Roche Diagnostics, Mannheim, Germany). The cycling conditions were designed according to the manufacturer's guidelines. Each cDNA sample was run in triplicate, and gene expression was normalized to the amount of glyceraldehyde-3-phosphate dehydrogenase on the same sample.

Western blotting. Cells were collected $72 \mathrm{~h}$ after transfection and lysed in mammalian cell lysis buffer and then Western blot analysis was performed with the use of conventional protocols. Cells were washed four times with PBS containing $1 \mathrm{mmol} / \mathrm{l}$ phenylmethylsulfonyl fluoride and scraped from dishes. Cell pellets were then lysed in cold TNT buffer $(20 \mathrm{mmol} / \mathrm{l}$ Tris-HCl, pH 7.4, 200 mmol/1 NaCl, 1\% Triton X-100, 1 mmol/l phenylmethylsulfonyl fluoride, and $1 \%$ aprotinin) for $30 \mathrm{~min}$. The lysates were transferred to new tubes and centrifuged at $4^{\circ} \mathrm{C}$. Proteins were separated in $10 \%$ SDS-PAGE gel followed by transfer to a membrane. After blocking in PBS $0.1 \%$ Tween $5 \% \mathrm{BSA}$ at room temperature for $1 \mathrm{~h}$, membranes were incubated with primary antibody overnight at $4^{\circ} \mathrm{C}$. The antibodies and dilutions used included anti-VEGFR3 and antiß-actin. After washes and incubation with secondary antibody the bound antibody was visualized on autogradiography film 


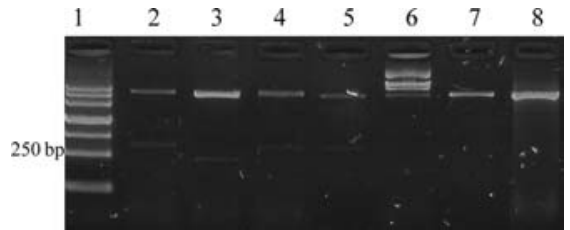

Figure 1. Identification of recombinant by restricted endnuclease digestion. Lane 1, 2000 bp DNA marker; 2, pSUPER-siRNA1/VEGFR3 was cut by the double restriction enzyme EcoRI and Hind III, and formed a 281-bp small fragment; 3 , pSUPER vector was cut by the double restriction enzyme EcoRI and Hind III, small fragment is 248 bp; 4, pSUPER-siRNA2/VEGFR3 was cut by the double restriction enzyme EcoRI and Hind III, small fragment is $281 \mathrm{bp} ; 5$, pSUPER-siRNA2/VEGFR3 was cut by the double restriction enzyme EcoRI and Hind III, small fragment is $281 \mathrm{bp} ; 6$, pSUPER vector was not cut by restriction enzyme; 7, pSUPER vector was cut by the restriction enzyme Hind III, no small fragment formed; 8, pSUPER-siRNA2/VEGFR3 was cut by the restriction enzyme $\mathrm{Bgl}$ II, lost the digestion site, no small fragment formed.

according to the manufacturer's protocol. Detection by enzymelinked chemiluminescence was performed according to the manufacturer's protocol. After film exposure with X-ray, the pictures were scanned and preserved as documents and the output of gray scale was quantified by Molecular Analysis software.

Murine xenograft model. Male BALB/c athymic nude mice, 4-6 weeks old, were injected subcutaneously in the right flank with $2 \times 10^{6}$ LoVo cells of PBS in $200 \mu 1$. After 3 weeks, tumor volume reached $68.4 \pm 35.2 \mathrm{~mm}^{3}$ and five mice were randomized for therapy into two experimental groups, siRNA treatment group and control group. Mice were anesthetized, and $50 \mu \mathrm{g}$ of pSUPER-siRNA2/VEGFR3 or pSUPER in $20 \mu \mathrm{l}$ of sterile $0.9 \% \mathrm{NaCl}$ solution were directly injected into the tumor mass. Tumor diameters were measured at regular intervals with calipers, and the tumor volume in $\mathrm{mm}^{3}$ was calculated by the formula, volume $=($ width $) 2 \times$ length $/ 2(15)$. The observations were terminated after 4 weeks from the day of vector injection. Animals were sacraficed and tumors were immediately frozen in dry ice or fixed in formalin. All animal experiments were carried out according to the University guidelines.

Immunohistochemical staining for VEGFR3 expression. The tumors from pSUPER-siRNA2/VEGFR3 or pSUPER injected mice were put into optimal cutting temperature compound, and 6-8- $\mu \mathrm{m}$ thick sections were cut. Immunohistochemical determination of VEGFR3 was also performed as described previously (16). VEGFR3, positive endothelial cell density was determined following a standard method for determination of microvessel density (10) as follows. The 5 most stained areas (i.e., the so-called hot-spots) within a section were selected for determination of VEGFR3-positive endothelial cell density; and microvessels highlighted with the anti-VEGFR3 antibody were counted under light microscopy with a 200 -fold magnification. Because some vessels, especially lymphatic vessels, may have collapsed, all the spots highlighted with the VEGFR3 antibody that were not identified as tumor cells were counted. The average counts were recorded as the VEGFR3-positive endothelial cell density for each case.

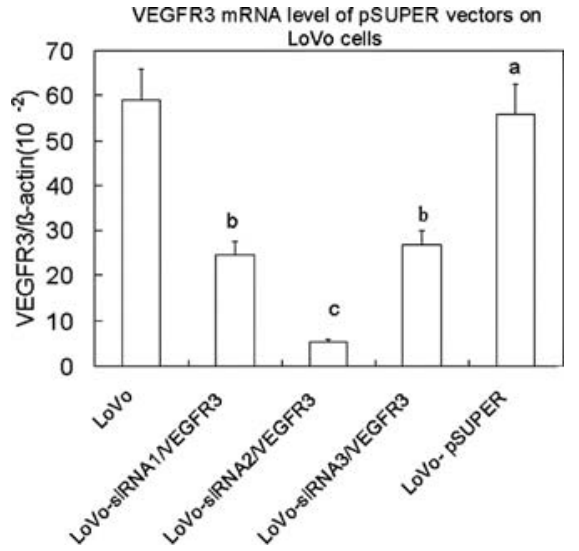

Figure 2. pSUPER-siRNA/VEGFR3 inhibits the expression of VEGFR3 in LoVo cells. Quantitative real-time RT-PCR indicated that pSUPERsiRNA2/VEGFR3 markedly reduced the level of VEGFR3 mRNA. Values are a concentration of VEGFR3 mRNA in LoVo cells, which were tansfected recombinant vectors or a naked vector. Values are mean \pm SD from five samples per group. LoVo cells without treatment were used as an internal control $\left({ }^{\mathrm{a}, \mathrm{b}} \mathrm{P}>0.05 ;{ }^{\mathrm{c}} \mathrm{P}<0.05\right)$.

Statistical analysis. All results are expressed as means \pm SD. The significance of the data was determined by Student's t-test for all studies. $\mathrm{P}<0.05$ was deemed significant. All statistical analyses were done using SPSS 15.0 software.

\section{Results}

Inhibition of VEGFR3 expression by expression of SiRNA in colon tumor cells. Three VEGFR3 siRNA expressing plasmids (plasmids 1,2, and 3) were constructed using the pSUPER vector. As shown in Fig. 1, the recombinant eukaryotic expressor vector pSUPER-siRNA/VEGFR3 was cut by the double restriction enzyme EcoRI and Hind III. The positive clone obtained is a 281-bp fragment and negative clone is 248 bp. We constructed three stably transfected pSUPERsiRNA/VEGFR3-LoVo cells, which were stably transfected with pSUPER-siRNA/VEGFR3 vectors or pSUPER vectors, respectively. The VEGFR3 protein levels were measured by quantitative real-time PCR and Western blot analysis. As shown in Fig. 2, the mRNA levels of VEGFR3 were analyzed by quantitative real-time RT-PCR after transfected for $48 \mathrm{~h}$. We observed significant inhibition of VEGFR3 mRNA expression in pSUPER-siRNA/VEGFR3 cells compared with controls in four cell lines as measured by qRT-PCR, pSUPERsiRNA1/VEGFR3 (24.6 \pm 15.8$)$, pSUPER-siRNA2/VEGFR3 (5.4 \pm 12.6$)$, and pSUPER-siRNA3/VEGFR3 (26.7 \pm 11.3$)$. The inhibition reached statistical significance $(\mathrm{P}<0.05)$. Whereas the slight inhibition observed in control cells $(55.8 \pm 14.6)(\mathrm{P}>0.05)$ by Western blot analysis showed a significant decrease in VEGFR3 protein levels in the pSUPER-siRNA2/VEGFR3 constructs (Fig. 3). It was shown that the pSUPER-siRNA2/ VEGFR3 was the most effective at silenced VEGFR3 expression in these recombination vectors. Therefore pSUPER-siRNA2/VEGFR3 was used in the subsequent experiments.

Effects of VEGFR3 siRNA on tumor cell proliferation. The biological effects of the VEGFR3 siRNA were first determined using cell proliferation assays. As shown in Fig. 4, the growth 


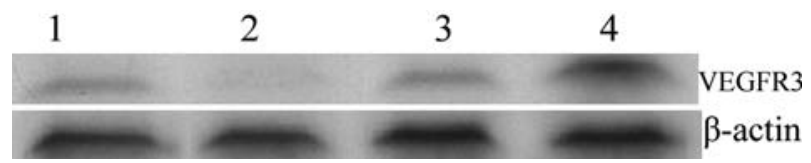

Figure 3. The effects of pSUPER-siRNA/VEGFR3 on the level of VEGFR3 protein were determined by Western blotting. $\beta$-actin was used as an internal marker. Lane 1, pSUPER-siRNA1/VEGFR3; 2, pSUPER-siRNA2/VEGFR3; 3, pSUPER-siRNA3/VEGFR3; 4, pSUPER.

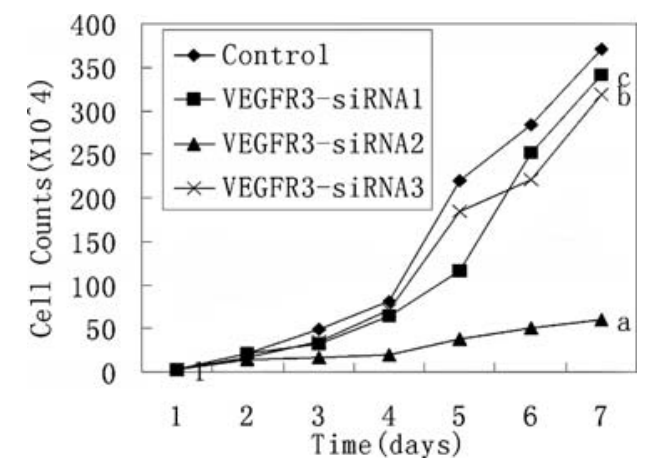

Figure 4. Effects of VEGFR3 siRNA on tumor cell proliferation as measured by the MTT assay. ${ }^{\mathrm{a}} \mathrm{P}<0.001,{ }^{\mathrm{b}} \mathrm{P}>0.05,{ }^{\mathrm{c}} \mathrm{P}>0.05$.

curves showed that the pSUPER-siRNA2/VEGFR3 cell proliferation was significantly silenced at days 3 and 4 ( $\mathrm{P}<0.001$, compared with the other group), whereas there was little difference in the other cells lines $(\mathrm{P}>0.05)$.

Effects of VEGFR3 siRNA on primary tumor growth in vivo. As shown in Fig. 5, pSUPER group cells grew rapidly. At the time of sacrifice, tumors in the control group had a volume of $572.5 \pm 43.1 \mathrm{~mm}^{3}$, which was 8.4 -fold larger than the starting volume, whereas in the pSUPER-siRNA2/VEGFR3 group, the tumors had a volume of $313.2 \pm 32.6 \mathrm{~mm}^{3}$, which means it increased only 4.4-fold over the starting volume. In contrast, tumor growth was significantly delayed $(\mathrm{P}<0.001)$ in the group of pSUPER-siRNA2/VEGFR3.

Effect of VEGFR3 siRNA on tumor lymphangiogenesis and angiogenesis. Tumor tissue from mice was excised and subjected to immunohistochemical staining. We examined the effect of VEGFR3 gene silencing on lymphangiogenesis and angiogenesis in the pSUPER-siRNA2/VEGFR3 group and the pSUPER control group by immunohistochemical analysis using anti-VEGFR3 antibody. Figs. 6 and 7 show these results as well as quantitative data from the microlymphatic and microvessel density analyses. The microvascular density (MVD) values (per 200x field) of subcutaneous tumors in pSUPER control and pSUPERsiRNA2/VEGFR3 cell lines were 4.8 $43.9,13.6 \pm 5.6$, $(\mathrm{P}<0.05)$ respectively. These data suggest that inhibition of VEGFR3 in primary tumors by VEGFR3 siRNA leads to decreased lymphangiogenesis and angiogenesis.

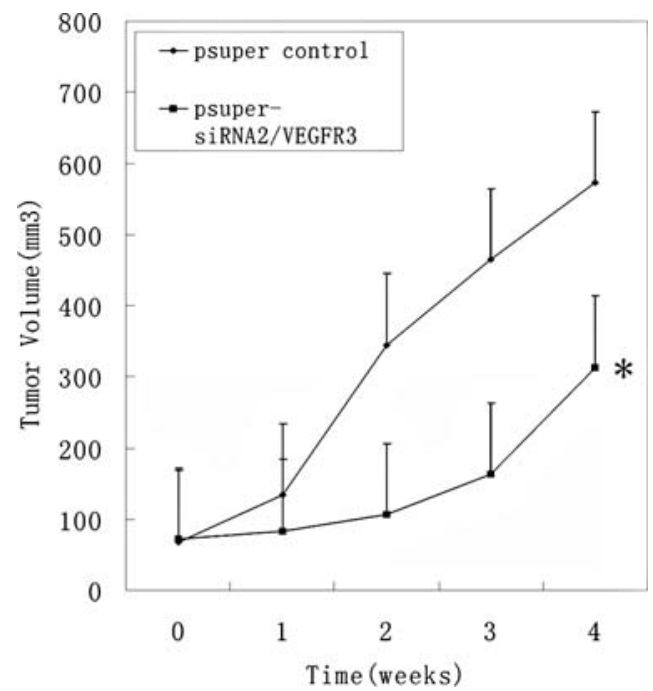

Figure 5. Effects on tumor growth of xenograft tumors. Each point represents the mean volume $\pm \mathrm{SD}$ on the $\mathrm{X}$-axis. Point 1 indicates the day of DNA injection; point 4 indicates when mice were sacrificed. ${ }^{*} \mathrm{P}<0.001$, VEGFR3 siRNA group vs control group.

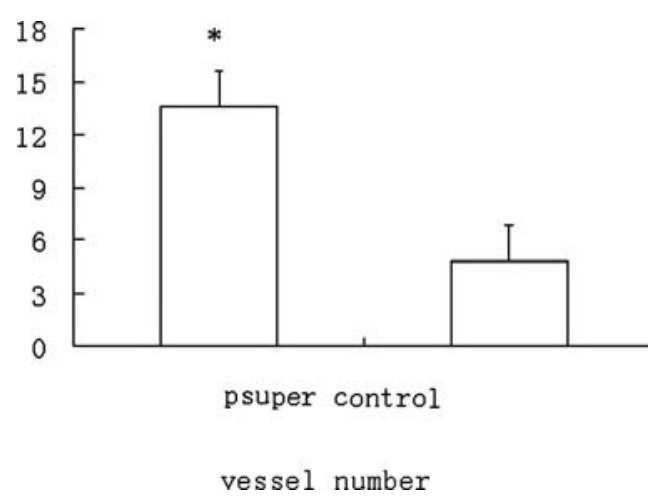

Figure 6. Effects of VEGFR3 siRNA on tumor cell angiogenesis in vitro. ${ }^{*} \mathrm{P}<0.001$

\section{Discussion}

The vascular endothelial growth factor receptor 3 (VEGFR3) gene is essential for the remodeling and maturation of embryonic blood capillaries and the lymphatic endothelium, fenestrated blood capillaries of some adult organs continue to express low amounts of VEGFR3 (15-17). Some studies found no relationship between them $(18,19)$. Furthermore, Padera et al (20) has shown that inhibiting VEGFR3 is not effective in treating lymph node metastasis after cells have arrived in the lymph node in this tumor model, but VEGFR3 overexpression of tumor cells, in experimental tumor models, resulted in intratumoral and peritumoral lymphangiogenesis and increased metastasis to the regional lymph nodes $(4,5)$. Data on animal models also suggested that VEGFR3 binds VEGF-C and -D and is essential for the development of the lymphatic vasculature $(6,7,21-23)$. When VEGFR3 is present on tumor cells or blood vessels, inhibiting VEGFR3 under similar conditions may lead to tumor suppression and reduce tumor burden in lymph nodes (24). 

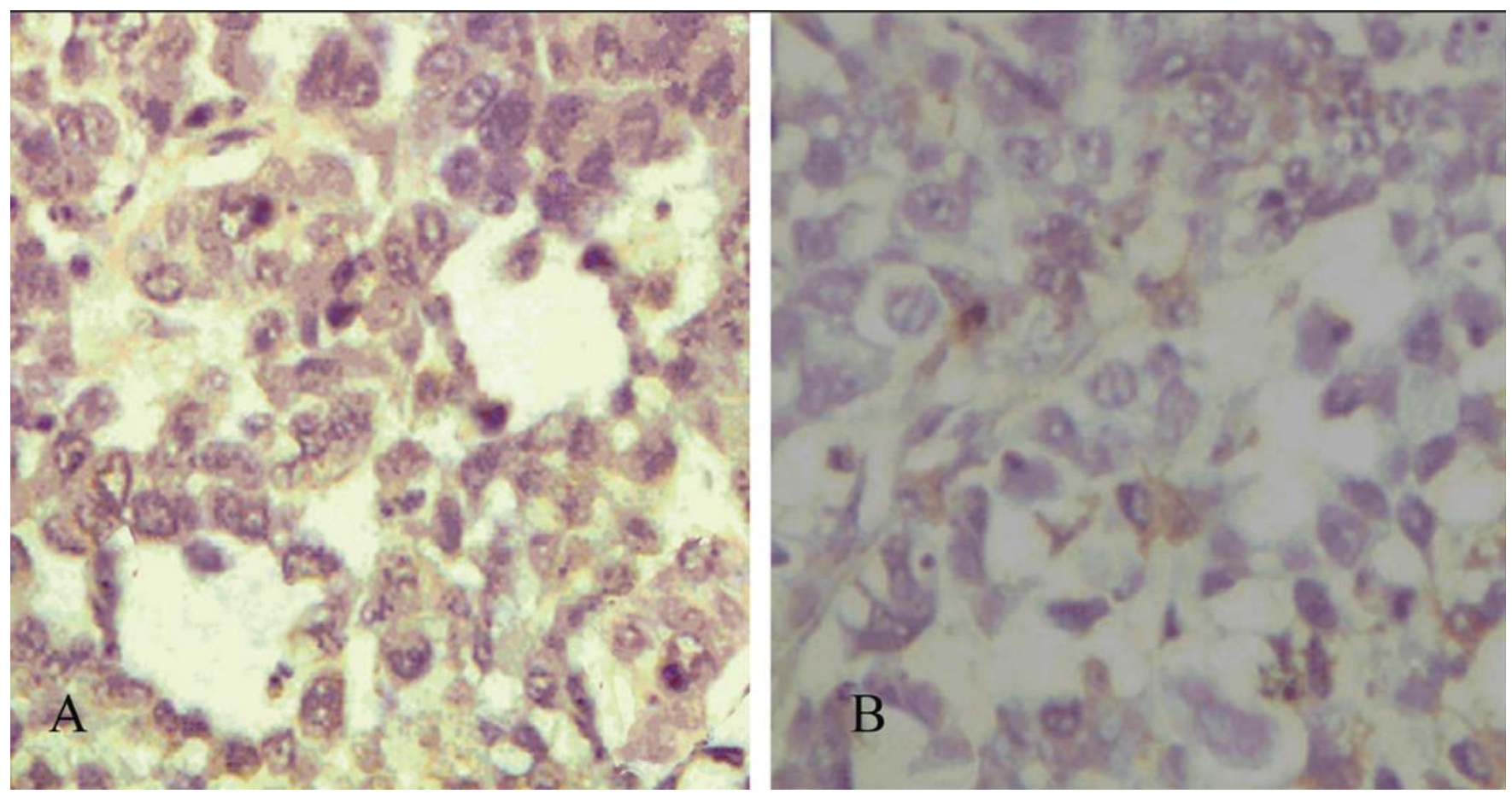

Figure 7. Immunohistochemistry for vascular endothelial growth factor receptor (VEGFR3) in tumors. VEGFR3-positive microvessels increased in the control group (A) compared with those in the VEGFR3-siRNA group (B) (original magnification x200).

RNA interference can induce post-transcriptional gene silencing through RNA-RNA binding and transcriptional gene silencing through RNA-DNA binding. Transcriptional gene silencing refers to siRNA molecules that hinder production of mRNA from DNA before gene transcription, by modifying chromosomal DNA and histones. This protocol has been used against several cancer targets, either as synthetic RNA oligo-nucleotides $(4,5)$ or as plasmid-encoded shRNA $(6,25)$, which are expected to be efficiently transcribed and subsequently processed to yield the mature, active form of siRNA. Our study showed that in VEGFR3 siRNA stable transfection of the colorectal cancer LoVo cell lines, VEGFR3 mRNA and protein production were downregulated. In contrast, VEGFR3 expression was unchanged in the controls. This experiment clearly showed that VEGFR3 siRNA inhibited the tumor growth of colorectal tumor and possibly blocked the VEGF-C, -D/VEGFR3 pathway to decrease lymphangiogenesis and metastasis to distant lymphatic nodes.

Therefore, it was also demonstrated that a single injection of pSUPER-siRNA2/VEGFR3 reduced the rate of growth of LoVo-derived xenografts and, most importantly, it had a lasting effect on tumor development, being effective for at least 14 days. However, the effective time of this siRNA expression vector-mediated RNA interference is still unclear and needs to be studied further.

In summary, the present study showed that RNA interference was an effective tool to inhibit VEGFR3 expression in colon tumor cells and inhibition of VEGFR3 had an effect on primary tumor growth. Based on these studies, we believe that down-regulation of VEGFR3 expression by siRNA provides a therapeutic strategy for inhibiting tumor growth and metastasis and for enhancing the survival of patients with colon cancer.

\section{Acknowledgements}

We are grateful to our colleagues in the Medical Laboratory, Lanzhou Regime General Hospital of PLA, for their excellent technical support.

\section{References}

1. Jemal A, Siegel R, Ward E, Murray T, Xu J and Thun MJ: Cancer statistics, 2007. CA Cancer J Clin 57: 43-66, 2007.

2. Alitalo K and Carmeliet P: Molecular mechanisms of lymphangiogenesis in health and disease. Cancer Cell 1: 219-227, 2002.

3. Pepper MS, Tille JC, Nisato R and Skobe M: Lymphangiogenesis and tumor metastasis. Cell Tissue Res 314: 167-177, 2003.

4. Karpanen T, Egeblad M, Karkkainen MJ, Kubo H, Ylä-Herttuala S, Jäättelä M and Alitalo K: Vascular endothelial growth factor C promotes tumor lymphangiogenesis and intralymphatic tumor growth. Cancer Res 61:1786-1790, 2001.

5. Mattila MM, Ruohola JK, Karpanen T, Jackson DG, Alitalo K and Härkönen PL: VEGF-C induced lymphangiogenesis is associated with lymph node metastasis in orthotopic MCF-7 tumors. Int J Cancer 98: 946-951, 2002.

6. Stacker SA, Caesar C, Baldwin ME, et al: VEGFD promotes the metastatic spread of tumor cells via the lymphatics. Nat Med 7: $151-152,2001$

7. Skobe M, Hawighorst T, Jackson DG, et al: Induction of tumor lymphangiogenesis by VEGF-C promotes breast cancer metastasis. Nat Med 7: 192-198, 2001.

8. He Y, Kozaki K, Karpanen T, Koshikawa K, Yla-Herttuala S Takahashi T and Alitalo K: Suppression of tumor lymphangiogenesis and lymph node metastasis by blocking vascular endothelial growth factor receptor-3 signalling. J Natl Cancer Inst 5: 819-825, 2002

9. Pytowski B, Goldman J, Persaud K, et al: Complete and specific inhibition of adult lymphatic regeneration by a novel VEGFR-3 neutralizing antibody. J Natl Cancer Inst 97: 14-21, 2005. 
10. He Y, Rajantie I, Pajusola K, et al: Vascular endothelial cell growth factor receptor 3-mediated activation of lymphatic endothelium is crucial for tumor cell entry and spread via lymphatic vessels. Cancer Res 65: 4739-4746, 2005.

11. Nilsson I, Rolny C, Wu Y, et al: Vascular endothelial growth factor receptor-3 in hypoxia-induced vascular development. FASEB J 18:1507-1515, 2004.

12. Valtola R, Salven P, Heikkilä $P$, et al: VEGFR-3 and its ligand VEGF-C are associated with angiogenesis in breast cancer. Am J Pathol 154:1381-1390, 1999.

13. Kubo H, Fujiwara T, Jussila L, et al: Involvement of vascular endothelial growth factor receptor-3 in maintenance of integrity of endothelial cell lining during tumor angiogenesis. Blood 96: 546-553, 2000

14. Elbashir SM, Harborth J, Weber K and Tuschl T: Analysis of gene function in somatic mammalian cells using small interfering RNAs. Methods 26: 199-213, 2002.

15. Dumont DJ, Jussila L, Taipale J, et al: Cardiovascular failure in mouse embryos deficient in VEGF receptor-3. Science 282: 946-949, 1998

16. Chen F, Takenaka K, Ogawa E, Yanagihara K, Otake Y, Wada H and Tanaka F: Flt-4-positive endothelial cell density and its clinical significance in non-small cell lung cancer. Clin Cancer Res 10: 8548-8553, 2004.

17. Partanen TA, Arola J, Saaristo A, et al: VEGF-C and VEGF-D expression in neuroendocrine cells and their receptor, VEGFR-3, in fenestrated blood vessels in human tissues. FASEB J 14 2087-2096, 2000.

18. Shida A, Fujioka S, Kobayashi K, Ishibashi Y, Nimura H, Mitsumori N and Yanaga K: Expression of vascular endothelial growth factor (VEGF)-C and -D in gastric carcinoma. Int J Clin Oncol 11: 38-43, 2006.
19. Kitadai Y, Kodama M, Cho S, et al: Quantitative analysis of lymphangiogenic markers for predicting metastasis of human gastric carcinoma to lymph nodes. Int J Cancer 115: 388-392, 2005.

20. Padera TP, Ancukiewicz M, Hoshida T, Fukumura D and Jain RK: Anti-VEGFR-3 therapy and lymph node metastasis. Cancer Res 67: 5055, 2007.

21. He XW, Yu X, Liu T, Yu SY and Chen DJ: Vector-based RNA interference against vascular endothelial growth factor-C inhibits tumor lymphangiogenesis and growth of colorectal cancer in vivo in mice. Chin Med J 121: 439-444, 2008.

22. Wang SC, Liu H, Ren LF, Pan YF and Zhang YD: Inhibiting colorectal carcinoma growth and metastasis by blocking the expression of VEGF using RNA Interference. Neoplasia 10: 399-407, 2008

23. Ishikawa M, Kitayama J, Kazama S and Nagawa H: Expression of vascular endothelial growth factor C and D (VEGF-C and D) is an important risk factor for lymphatic metastasis in undifferentiated early gastric carcinoma. Jpn J Clin Oncol 33: 21-27, 2003

24. Laakkonen P, Waltari M, Holopainen T, et al: Vascular endothelial growth factor receptor 3 (VEGFR-3) is involved in tumor angiogenesis and growth. Cancer Res 67: 593-599, 2007.

25. Onogawa S, Kitadai Y, Amioka T, et al: Expression of vascular endothelial growth factor (VEGF)-C and VEGF-D in early gastric carcinoma: correlation with clinicopathological parameters. Cancer Lett 226: 85-90, 2005 